\title{
EL RAZONAMIENTO ORACULAR Y LOS LÍMITES DE LA CREENCIA JUSTIFICADA EN LOS ARGUMENTOS TIPO GETTIER ACERCA DEL CONOCIMIENTO.
}

\author{
ALEJANDRO MATUS \\ alejandromatusunfe020@gmail.com
}

\begin{abstract}
Resumen. A través de un análisis lógico, mostramos cómo los casos Gettier, presentados como contraejemplos a la concepción del conocimiento como creencia verdadera justificada, no son consistentes, al ser una variación del razonamiento de tipo oracular, falacia lógica en la cual son embrolladas las proposiciones interpretadas, que contienen una constante lógica, con proposiciones formadas por variables lógicas; son confundidas la unicidad con la mera existencia, las condiciones necesarias y suficientes con condiciones necesarias pero no suficientes; y hay sesgos cognitivos, por ejemplo, el de descuido de las frecuencias de base en la argumentación.
\end{abstract}

\section{PALABRAS CLAVE: FALACIAS_EPISTEMOLOGÍA_GETTIER_PREJUI- \\ CIOS_TVERSKY_CREENCIA_JUSTIFICACIÓN_NECESARIO-SUFICIENTE_DEDUC- CIÓN-EXPERIENCIA_LÓGICA_CUANTIFICADORES_FRECUENCIAS-BASE.}

Contra la concepción del conocimiento como creencia verdadera justificada, Edmund L. Gettier ha propuesto dos casos como contraejemplos. Ello ha tenido fuerte repercusión entre la comunidad de los estudiosos de la Teoría del Conocimiento, donde, incluso, se ha visto multiplicada la estipulación de contraejemplos de tal clase. Existe difundida la idea de que ellos son razonamientos lógicamente válidos, que hacen necesario corregir la caracterización del conocimiento mencionada, ya sea modificando o bien ampliando las condiciones para describirlo (cf. Dretske 1971; Goldman 1976; Nozick 1981, pp.178-180; Lycan 2006).

En el presente artículo realizamos un análisis para mostrar que esos contraejemplos tienen fallas lógicas que impiden el que sean válidos. Fallas que consisten básicamente en una forma de razonamiento muy semejante a la de muchos oráculos y predicciones astrológicas, donde se confunden proposiciones interpretadas que contienen constantes lógicas con proposiciones integradas por variables lógicas sin interpretar; cuantificadores lógicos de unicidad con cuantificadores de mera existencia; condiciones necesarias y suficientes con condiciones necesarias, pero no suficientes; y las tasas de base (cf. Tversky-Kahneman 1982) pertinentes para la argumentación.

Para mostrar tal semejanza estructural, primero analizamos en qué consiste el razonamiento oracular y su errada estructura lógica. Después, aplicamos los resultados a los dos casos expuestos por Gettier, que dependen de deducciones lógicas. Y a cómo tales consecuencias se aplican también a los casos generados posteriormente por otros autores, referidos al conocimiento empírico. Deteniéndonos en la manera en que se pueden agotar las formas de justificar una creencia (la deducción lógica o la experiencia directa o indirecta). Finalmente, enunciamos en qué consiste en general la estructura profunda y falaz del procedimiento.

\section{El razonamiento oracular.}

Heródoto cuenta:

Creso (rey de Lidia) les ordenó (a sus emisarios) que preguntaran a los oráculos si debía emprender la guerra contra los persas. 
El oráculo de Delfos le dijo

Si emprendes la guerra contra los persas, destruirás a un gran imperio ... Creso, quedó plenamente seguro de destruir el reino de Ciro (el rey persa) ... Y de ahí que, al recibir un ambiguo vaticinio que consideró le era favorable, se lanzara con su ejército contra el territorio persa.

Creso fue derrotado.

Conforme al oráculo, había puesto fin a un gran imperio: el suyo propio.

Ciro le permitió enviar otros emisarios para

Preguntar al dios (Apolo) si no se avergonzaba de haberle instigado con sus vaticinios a entrar en guerra con los persas con la promesa de que destruiría el poderío de Ciro ... Se cuenta que la Pitia se pronunció en estos términos: ... Creso se queja sin razón, pues Loxias le predijo que, si entraba en guerra con los persas, pondría fin a un gran imperio. Pero, ante esta respuesta, tenía que haber enviado a preguntar - para adoptar una decisión acertada - si se refería a su imperio o al de Ciro. Y si no entendió la respuesta ni pidió explicaciones, que se considere a sí mismo responsable. (Heródoto Libro I, pp. 53-54, 75, 86, 90-91)

Al final, Creso aceptó esta réplica.

\section{La estructura lógica del razonamiento oracular.}

Este vaticinio es un ejemplo claro de gran número de predicciones oraculares y astrológicas, con una estructura lógica del siguiente tipo:

a) El profeta emite una proposición ambigua, es decir, una proposición general que admite más de una opción como especificación.

b) Alguien, normalmente el destinatario de la predicción, especifica tal proposición, dándole un sentido unívoco, habitualmente correspondiente a antecedentes personales, prejuicios y esperanzas.

c) Si ocurre en los hechos tal opción en particular, el oráculo clama haber acertado. Si no ocurre ella, el oráculo evade su responsabilidad achacando una errónea especificación al interesado. El adivino afirma que lo que quiso decir fue la opción en particular que en realidad ocurrió, la cual, desde luego, es consistente con la generalidad de la predicción.

Esto lo podemos analizar en términos lógico formales:

a') El augur emite una función proposicional, con una variable x que puede tomar más de un valor; y no una sentencia interpretada, específica, que le de a $\mathrm{x}$ un valor constante. El adivino maneja su función proposicional sin especificar a qué es igual x. Sólo afirma Fx. b') Es el interesado el que le da un valor a $x$, normalmente sin darse cuenta que le han dejado a él tal papel, dando por hecho que tal especificación interpretativa va implícita en la emisión del profeta. Así, el interesado cree que le ha sido proporcionada no Fx, sino, por ejemplo, $\mathrm{Fa}$, con $\mathrm{x}=\mathrm{a}$.

c') Si ocurre $\mathrm{Fa}$, se da por acertado el oráculo $(\mathrm{Fx} \equiv \mathrm{Fa})$, tanto por el que pregunta como por el adivino. Si ocurre $x \neq a$, el augur atribuye el error al que pregunta; $y$ si, además, $x=b$, $\mathrm{y}$, por lo tanto, $\mathrm{Fb}$, afirma que eso fue lo que quiso decir $(\mathrm{Fx} \equiv \mathrm{Fb})$ y por lo tanto acertó. Con frecuencia quien pregunta acaba por aceptar esa respuesta. 
La estructura de la falacia consiste en emitir una función proposicional, pero considerarla (indebidamente desde un punto de vista lógico) en sí misma como una fórmula interpretada, más específica de lo que en realidad es. Aprovechar que la función tiene más de una opción de concretización, pero tratarla como si en sí misma ella fuera una sola opción. Permitir que se le de un valor específico y no objetar tal valor, hasta que ocurra el hecho concreto que lo hace ser verdadero o falso.

En caso de ser verdadero, se da por validada la función proposicional.

En caso de falsedad, se deja que la falsedad recaiga en la primera fórmula ya tomada como opción particular. Pero se recurre de manera implícita a una segunda opción específica, la que corresponde al hecho que realmente ocurrió. Y se cree, o se afirma, que al emitir la función proposicional lo que se quiso decir fue la nueva determinación.

Al inicio, se considera que tienen la misma validez la función proposicional y su primera fórmula especificada. Posteriormente, se pasa a tomar como idénticas a la misma función proposicional y a una segunda fórmula, especificada de manera diferente a la primera, y contradictoria con ésta.

\section{La argumentación de Gettier. Primer caso.}

A continuación, aplicaremos lo dicho al razonamiento de Gettier.

Él se pregunta famosamente "¿Es conocimiento la creencia verdadera justificada?" (Gettier, 1963), al abordar una concepción de diversos epistemólogos (cf. Platón, Menón 97a98 b y Teeteto 200d-210c; Ayer, 1956, p. 34; Chisholm, 1957, p. 16), según la cual las condiciones necesarias y suficientes para que alguien conozca una proposición dada son:

1) La proposición $P$ es verdadera;

2) $S$ cree que $P$; y

3) S está justificado en creer que P.

Gettier sostiene que tal concepción es falsa, al poder formular contraejemplos al respecto.

Así, plantea lo siguiente:

Supóngase que Smith y Jones presentaron una solicitud para determinado empleo. Y supóngase que Smith tiene evidencia sólida de la siguiente proposición conjuntiva:

(d) Jones es el hombre que obtendrá el empleo, y Jones tiene diez

monedas en su bolsillo. ...

La proposición (d) implica:

(e) El hombre que obtendrá el empleo tiene diez monedas en su bolsillo.

Vamos a suponer que Smith ve la implicación que va de (d) a (e), y acepta

(e) basado en (d), para la cual tiene evidencia sólida. En este caso, Smith está claramente justificado en creer que (e) es verdadera. Pero imagínese, además, que aunque Smith no lo sepa, él mismo, no Jones, obtendrá el trabajo. Y también que, aunque Smith no lo sepa, él mismo tiene diez monedas en su bolsillo. La proposición (e) es entonces verdadera, aunque la proposición (d), desde la cual Smith infiere (e), es falsa. En nuestro ejemplo, por tanto, todo lo que sigue es verdadero:

(i) (e) es verdadera

(ii) Smith cree que (e) es verdadera

(iii) Smith está justificado en creer que (e) es verdadera.

Pero es igualmente claro que Smith no sabe que (e) es verdadera; puesto que (e) es verdadera en virtud del número de monedas en el bolsillo de Smith, aun cuando Smith no sepa cuantas monedas hay en el bolsillo de 
Smith, y basa su creencia en (e) sobre la cantidad de monedas en el bolsillo de Jones, de quien falsamente cree que será la persona que obtendrá el trabajo. (Gettier, 2013)

Analicemos esa propuesta de contraejemplo:

La proposición (d) corresponde a una expresión del tipo

$\exists \mathrm{x}: \mathrm{x}=\mathrm{j} \wedge \mathrm{T} \mathrm{x} \wedge \mathrm{Mx}$.

Donde $\mathrm{j}=\mathrm{Jones} ; \mathrm{T}=$ Conseguirá el trabajo; $\mathrm{M}=$ Tiene diez monedas en su bolsillo.

Aquí $\mathrm{x}$ ha adoptado el valor de una constante individual, que tiene siempre el valor de $\mathrm{j}$. Se refiere a un individuo específico que cumple con las condiciones estipuladas en la descripción. Si bien (e) se deriva de (d), el argumento de Gettier hace a un lado el que (e) es una función proposicional no específica. Únicamente cuando se ha optado por una constante especifica con $\mathrm{x}=\mathrm{a}, \mathrm{o} \mathrm{x}=\mathrm{b}, \mathrm{o} \mathrm{x}=\mathrm{s}, \mathrm{o} \mathrm{x}=\mathrm{j}$, etc., como en (d), se convierte en una fórmula particularizada, interpretada, a la que se puede asignar un valor de verdad o falsedad respecto a un sólo objeto concreto, a una sola opción.

Así, Gettier en (e) toma a x como si fuera una variable aún indeterminada, que puede adoptar valores distintos a j. Y deriva a partir de ello un nuevo valor de $\mathrm{x}$, cuando ocurre el caso de que "Smith" es el valor específico que hace verdadera a (e). Generando implícitamente una nueva proposición determinada (f). Y al hacer eso, en realidad está realizando una nueva operación, en la cual (e) $\rightarrow(\mathrm{f})$, por sustitución de la variable x con la nueva constante $s \neq j$. Siendo (f):

f) El hombre, Smith, que conseguirá el trabajo tiene diez monedas en su bolsillo.

Dando a x, "El hombre", el valor de "Smith".

En lugar de la expresión derivada de la original:

e') El hombre, Jones, que conseguirá el trabajo tiene diez monedas en su bolsillo.

Que da a la variable x, "El hombre", el valor de "Jones".

En otras palabras, se trata aquí de una confusión en los cuantificadores lógicos; de la diferencia entre "Existe uno y sólo uno" (que predica la existencia y la unicidad de un objeto, $\mathrm{y}$ exclusivamente puede ser, por ejemplo, $\mathrm{x}=\mathrm{j}$, que asigna a $\mathrm{x}$ un valor específico constante), y "Existe alguno" (que predica solamente la existencia, y puede ser $\mathrm{x}=\mathrm{a}$ o $\mathrm{x}=\mathrm{b}$ $\mathrm{o} x=\mathrm{j}$, etcétera).

Más exactamente, cabe formular a (d) de una manera sintácticamente válida como

d') $E ! x: x=j \wedge T j \wedge M j$.

Donde $\mathrm{E} !=$ Existe una y sólo una.

Como esta descripción incluye de manera lógico formal la afirmación de que el individuo posee de una manera exclusiva las características que se le atribuyen, y no las posee ningún otro individuo, además de la existencia se predica la unicidad, el que sólo existe un objeto tal que cumple con esas características (cf. Russell 1973).

Así tenemos, equivalentemente:

$\left.\mathrm{d}^{\prime}\right) \exists \mathrm{x} \forall \mathrm{y}(\mathrm{Hx} \wedge \mathrm{Tx} \wedge \mathrm{Mx} \wedge(\mathrm{Ty} \wedge \mathrm{My} \rightarrow \mathrm{y}=\mathrm{x}) \wedge \mathrm{x}=\mathrm{j})$

Es decir, existe un $x$ tal que $x$ es hombre $(H)$, que va a obtener el trabajo, y ese hombre tiene diez centavos en el bolsillo; y si cualquier hombre obtiene el trabajo, y ese hombre tiene diez centavos en el bolsillo ese hombre es Jones. 
La parte ligada $\forall \mathrm{y}(\mathrm{Ty} \wedge \mathrm{My} \rightarrow \mathrm{y}=\mathrm{x})$ deja fuera el que cualquier otro individuo distinto de Jones cumpla con la o las condiciones necesarias y suficientes expresadas por la proposición $\mathrm{P}$.

(d') es lo que cree saber Juan. Que si existe un hombre al que se le llame "y" (por ejemplo, "el hombre de cabello negro" o "el hombre de la camisa café" o "el hombre de ojos verdes" o "el hombre con diez monedas en el bolsillo"), y ese hombre obtiene el empleo, ese hombre es Jones $(\mathrm{T} y \rightarrow \mathrm{y}=\mathrm{x})$.

En su argumento, al implicar a (e), Gettier hace a un lado, válidamente, la condición necesaria $(\mathrm{Ty} \wedge \mathrm{My} \rightarrow \mathrm{x}=\mathrm{y})$. Mas usa "El hombre" como una variable que puede ser sustituida por cualquiera de un conjunto de constantes alternativas, por varias opciones, cuando en realidad en (d) ocurre una única opción, una y sólo una constante lógica definida.

Dicho de otra forma, Gettier sustituye, primero, lo particular por lo general. A una proposición específica $\mathrm{Fj}$ acerca de Jones la convierte en una proposición Fx acerca de un individuo posible que puede ser, o no, Jones, pero que viene del supuesto de que es Jones.

Pero luego a esta Fx, inadvertida y falazmente, como en el razonamiento oracular, la convierte en una nueva y diferente proposición Fs, ahora acerca de otra constante: Smith.

Lo que hace Gettier es darle un nuevo valor específico a la variable $\mathrm{x}$ a partir de la función proposicional (e), el de $\mathrm{x}=\mathrm{s}$, generando distraídamente una nueva proposición (f) distinta de (d) y de (e), contradictoria de (d), en un paso no justificado.

Y esta opción (f), mas no la que Smith cree conocer, (e), es la opción de fórmula específica, la proposición, que al final adopta Gettier, que es verdadera o falsa al asignarse el empleo, o no, solamente a Smith, a s; mientras que la opción más general (e) puede ser verdadera al asignarse el empleo tanto a Smith como a Jones, o a cualquier otra persona con diez monedas en el bolsillo. Es decir, tanto a Smith como a no Smith, a s como a $\neg$; a Jones, o a no Jones, a j como a $\neg$ j.

El sentido de (e) para la creencia de Smith siempre presupone a Jones. "El hombre que conseguirá el trabajo tiene diez monedas en su bolsillo" siempre significa "Jones" para Smith y por ello modalmente no es sustituible nunca por "Smith" o algún otro. La cuestión no es acerca de la mera proposición, sino de la creencia de Juan acerca de la proposición.

Es falso que Smith crea justificadamente que (e) sin la univocidad previa. Lo que Gettier realiza es un cambio de constante falaz respecto a $(\mathrm{d})$, formando a $(\mathrm{f})$; y confunde $a(e)$ con $(f)$. Por lo tanto, su Smith no está justificado lógicamente en creer que $\mathrm{P}$ es verdadero bajo cualquier opción de especificación.

En el caso de Creso, con cualquiera de las dos opciones que acontezca, los sacerdotes podrán decir que la pitonisa tuvo razón. Lo que ocurrió es que ella emitió una oración tipo (e), con una estructura de función proposicional no específica, ambigua, con más de una opción, sin asignarle un valor definid o a x. Y fue a continuación, implícitamente, sin darse cuenta, que de manera falaz Creso mismo sustituyó la x por la constante de su agrado ("Persia"); y después de los hechos, el oráculo la sustituyó por la constante "Lidia"; así como Gettier toma en (d) a la x por "Jones" y luego en (f) sustituye a partir de (e) la x por "Smith".

\section{Condiciones necesarias y suficientes.}

La condición "Uno y sólo uno" recalca la necesidad de plantear las condiciones necesarias y suficientes para especificar a ese uno determinado. Además de las condiciones generales necesarias y suficientes para que exista conocimiento, en el caso específico expuesto 
por Gettier, para hablar del conocimiento de Smith es necesario y suficiente que se cumplan las condiciones particulares de existencia y unicidad:

1) Jones es la persona que obtendrá el trabajo,

2) Existe una y sólo una persona que obtendrá el trabajo; y

3) Jones tiene diez monedas en el bolsillo.

Desde el punto de vista lógico del conocimiento en concreto que pueda tener Smith, son esas tres las condiciones necesarias y suficientes determinadas por (d) para que conozca que P. Cuando deduce (e) a partir de (d), suprime una de esas condiciones, la principal, la de $E ! x: x=j$; con lo que (e) ya no expresa las condiciones suficientes en este caso específico para que Smith crea que (d).

Cuando de las tres condiciones Gettier retoma únicamente dos en (e), y excluye la de $\mathrm{x}=$ Jones, sólo puede deducir de manera válida, no oracular, que Smith conoce que esas dos condiciones son necesarias, pero que no son suficientes para especificar quién obtendrá el trabajo. Pero Gettier razona erróneamente como si para Smith esas dos razones fueran suficientes.

De suponer que (1), (2) y (3) son razones necesarias y suficientes para afirmar que Smith cree P, pasa falazmente a suponer que (2) y (3) son razones necesarias y suficientes para afirmar que Smith cree la proposición P. Cuando lo único válido es concluir que (2) y (3) son razones necesarias, pero no suficientes (al faltar (1) con $\mathrm{x}=\mathrm{j}$ ) para afirmar que Smith cree P. Y así una de las condiciones necesarias para decir que Smith cree P no se ha cumplido. Es necesario para afirmar con verdad que Smith cree P, que $\exists x$ :Tx $\wedge$ Mx, pero no es suficiente. Es necesario también que $\mathrm{x}=\mathrm{j}$.

Entonces, Gettier, desde

Smith cree $\mathrm{P} \leftrightarrow(1),(2)$ y (3)

(o sea, P si y sólo si $\mathrm{E} ! \mathrm{x}: \mathrm{x}=\mathrm{j} \wedge \mathrm{Tx} \wedge \mathrm{Mx}$ ),

pasa implícitamente a

Smith cree $\mathrm{P} \leftrightarrow(2)$ y (3)

(viz., $\mathrm{P}$ si y sólo si $\exists \mathrm{x}: \mathrm{Tx} \wedge \mathrm{Mx}$ ).

Conservando erradamente el "si y sólo si".

Cuando lo justificado sintácticamente sólo es

Smith cree $\mathrm{P} \rightarrow(2)$ y (3)

(es decir, que $\mathrm{P}$ implica $\exists \mathrm{x}: T \mathrm{x} \wedge \mathrm{Mx}$ ).

Esto es, con la implicación simple, mas ya no con el si y sólo si.

Por ende, si Smith razona de una manera lógicamente coherente, no puede creer que (e) es verdadera si presupone aquellas razones necesarias como suficientes. O, si cree que con tal presuposición (e) es verdadera, entonces no está justificado lógicamente para creer tal cosa. Como sí lo está para creer que (e) es verdadera si presupone esas razones como necesarias, pero no suficientes.

En síntesis, Gettier emite (e), que tiene la estructura de una función proposicional, con base en (d) - con (d) como fórmula interpretada. Pero no ocurre la opción expresada por (d), sino la expresada por otra fórmula especificada (f) distinta. Subterráneamente, Gettier vuelve a especificar (e), ahora con base en el hecho que, según su planteamiento, realmente ocurre (lo expresado por la fórmula interpretada (f)). 
Y atribuye indebidamente la creencia en la verdad de esta nueva especificación (f) a la oración (e), la función proposicional de la que proviene, y no solamente, como lógicamente corresponde, a la nueva fórmula interpretada (f).

Es como si G, un aficionado a cocinar, quisiera aplicar la receta para un postre de un Chef reconocido que especificara:

d) Agregar canela (cf. "El imperio de Ciro va a caer"; o "Jones es el hombre que obtendrá el empleo y tiene diez monedas en su bolsillo").

Pero G no tuviera canela a su disposición en ese momento, y dedujera que como (d) implica la generalización:

e) Agregar una especia (cf. "Un gran imperio va a caer"; o "El hombre que obtendrá el empleo tiene diez monedas en su bolsillo"),

entonces puede

f) Agre gar pimienta, en tanto también es una especia (cf. "El imperio de Creso va a caer"; o "El hombre (Smith) que conseguirá el trabajo tiene diez monedas en su bolsillo)".

Y al hacerlo, el postre tuviera mal sabor. Y por lo tanto concluyera que la receta en realidad es mala, ... y no su forma de entender la lógica.

\section{El segundo caso de Gettier.}

\section{Dice Gettier:}

Vamos a suponer que Smith tiene evidencia sólida para la siguiente proposición:

(f) Jones es propietario de un Ford. ...

Smith tiene otro amigo, Brown, cuyo paradero desconoce totalmente. Smith selecciona tres nombres de lugares de manera completamente aleatoria y construye las tres proposiciones siguientes:

(g) o Jones es propietario de un Ford o Brown está en Boston;

(h) o Jones es propietario de un Ford o Brown está en Barcelona;

(i) o Jones es propietario de un Ford o Brown está en Brest-Lito vsk. ... Imagínese que Smith ... procede a aceptar (g), (h), e (i) sobre la base de (f). ... Por tanto, está completamente justificado en creer cada una de estas tres proposiciones. ... Pero imaginemos ahora que tenemos dos condiciones adicionales. Primero, Jones no es propietario de un Ford, sino que en la actualidad conduce un automóvil alquilado. Y segundo, que por la más pura coincidencia, y enteramente desconocido por Smith, el lugar mencionado en la proposición (h) resulta ser realmente el lugar donde está Brown. Si estas dos condiciones se dan, entonces Smith no sabe que (h) es verdadera, aun cuando: $(i)$ (h) es verdadera, (ii) Smith realmente cree que (h) es verdadera y (iii) Smith está justificado en creer que (h) es verdadera. (Gettier 2013)

En este caso, Gettier sólo escoge tres proposiciones específicas para sus disyunciones. Smith forma a partir de (f) tres sentencias de la forma (f) VX (con X una proposición variable), entre ellas (h), donde sustituye X por B: "Brown está en Barcelona".

Pero también se pueden formar infinidad de otras oraciones, sustituyendo X por otras sentencias, como, por ejemplo,

$\left.\mathrm{h}^{*}\right)$ : o Jones es propietario de un Ford o Brown NO está en Barcelona. 
Donde $X=\neg B$, o sea, se da $(f) \vee \neg B$. Y se puede creer justificadamente que todas esas proposiciones son verdaderas, al ser (f) verdadera. Después, ocurra B o $\neg B$, aunque (f) sea falsa, la fórmula proposicional (f) VX será siempre verdadera (pues B o $\neg \mathrm{B}$ agota por lógica al universo de opciones posibles), gracias a cualquiera de aquellas ocurrencias que resulte ser verdadera respecto a la variable X. Así, si bien Smith cree justificadamente que

h) o Jones es propietario de un Ford o Brown está en Barcelona,

al mismo tiempo cree justificadamente que

h*) o Jones es propietario de un Ford o Brown NO está en Barcelona.

Entonces, esté Brown o no esté Brown en Barcelona, cualquiera de esos dos hechos excluyentes entre sí (contradictorios entre sî) que acontezca hará verdadero a (f)VX.

Lo que es más, la generalidad de los autores parecen no haberse dado cuenta de que Gettier pudo haber escogido mejor la opción "No está en Barcelona", y su argumentación no necesitaría aducir "La más pura coincidencia", y tendría mucha mayor probabilidad de acertar al azar. Aunque con la misma falta de validez lógica.

Pasar de (f) a (f) VX es hacer una generalización tautológica válida, donde en $\mathrm{X}$ cabe cualquier proposición. Mas cualquiera que especifique a X y la haga verdadera, convirtiéndola en una proposición constante que hace verdadera a una nueva oración de la forma (f) VX, es elegida por Gettier como hace el oráculo, como si fuera la prevista para convertir en creencia verdadera justificada a (f) $V X$.

O bien Smith no cree que B (o que $\neg \mathrm{B}$ ) ocurra en los hechos, o sí lo cree. Pero entonces tiene una creencia injustificada, al juzgar que considerar que pueda acontecer cualquier cosa que se le ocurra le da el conocimiento concreto de algo. Así entraríamos a un sistema lógico donde cualquier cosa puede ser deducida, donde cualquier creencia puede ser justificada, un sistema inconsistente.

Smith tiene la idea general de que necesariamente una de esas proposiciones es verdadera y las otras falsas. Pero no de cuál es cuál. Y al especificar Gettier una de ellas post hoc, conforme a lo que haya ocurrido según su planteamiento de la realidad, está elaborando, a la manera oracular, una nueva proposición más específica (h), la cual no estaba originalmente ni en la oración (f) ni en su implicada (f) VX (de ésta, a continuación, se deriva (h), así como ( $\left.\mathrm{h}^{*}\right)$, o un número infinito de otras potenciales especificaciones que pueden convertirla en verdadera).

Gettier omite en este caso hacer explícita una acción intermedia, la de su paso hacia (f)VX, de la que después deduce (g), (h) e (i). Así tenemos que Smith cree que (f)VX es verdadera, de manera justificada, pero, asimismo, o bien

(ii') Smith realmente NO cree que (h) es verdadera porque Brown esté en Barcelona;

o, si lo hace,

(iii') Smith NO está justificado en creer que (h) es verdadera porque Brown esté en Barcelona.

Ya que en ambos casos también podría creer justificadamente y al mismo tiempo que $\left(h^{*}\right)$ también es verdadera porque Brown no esté en Barcelona. 
Por el planteamiento inicial, Smith no sólo cree en la disyunción, también cree que la condición que hace verdadera a la disyunción es únicamente la parte (f). Sin importar si la parte B es verdadera o falsa. El oráculo justifica: dijimos que alguna de las dos cosas iba a ocurrir y ocurrió. El oráculo no es unívoco, pero toma a la disyunción, aún sin especificar, como si fuera una proposición unívoca, como si fuera una y sólo una de sus especificaciones (adoptada a posteriori), la (h).

\section{Otros casos Gettier.}

Se afirma que

En los casos Gettier, la creencia verdadera justificada es inferida a partir de una creencia falsa justificada ... Sin embargo, ... existen ejemplos de casos Gettier que no necesitan incluir inferencia alguna. (Ichikawa 2018. Traducción propia)

Y:

Lo que distingue estos casos de los contraejemplos precedentes sobre la suficiencia es que, en ellos, no hay asunciones tácitas falsas identificables. (Lycan 2006, p. 157. Traducción propia)

Ejemplos de tales casos pueden ser los siguientes:

Preguntaba el hindú Dharmottara:

Imaginen que estamos buscando agua en un día caluroso. Repentinamente vemos agua, o eso pensamos. De hecho, no estamos viendo agua sino un espejismo, pero cuando alcanzamos el punto, tenemos suerte y encontramos agua justo ahí bajo una roca. ¿Podemos decir que tuvimos un genuino conocimiento del agua? La respuesta parece ser negativa, pues sólo tuvimos suerte. (Citado en Dreyfus 1997, p. 292. Traducción propia)

Por otra parte, el filósofo Pedro de Mantua planteaba:

Asúmase que Platón está cerca de usted y usted sabe que él está corriendo, pero erróneamente usted cree que él es Sócrates, de manera que usted cree firmemente que Sócrates está corriendo. Sin embargo, ocurre que Sócrates está corriendo de hecho en Roma; sin embargo, usted no conoce esto. (Citado por Boh 1985, p. 95. Traducción propia)

Chisholm presenta el siguiente caso:

Una persona considera que hay una oveja en el campo y lo hace en condiciones que son tales que, cuando en esas condiciones una persona considera que hay una oveja en el campo, entonces es evidente para esa persona que hay una oveja en el campo. La persona, sin embargo, ha confundido a un perro con una oveja y por lo tanto lo que ve no es una oveja en absoluto. Sin embargo, sucede que hay una oveja en otra parte del campo. Por lo tanto, la proposición de que hay una oveja en el campo será tanto verdadera como evidente y también será una que la persona acepte. Pero la situación no justifica que digamos que la persona sabe que hay una oveja en el campo. (Chisholm 1977, p. 93, Traducción propia)

En todos estos casos, lo que se cree, la proposición (1) que se formula, es referente a una ubicación precisa, y a un objeto preciso que se está considerando (el espejismo, Platón o el perro); y nuestro posible conocimiento es referido solo a esa ubicación y a ese objeto. 
En lo cual, por el planteamiento mismo, se nos confirma que tuvimos una creencia no verdadera.

Se está razonando de una forma oracular si se quiere hacer efectiva la idea de que se tuvo una creencia justificada, con una nueva proposición (2), al generalizar hacia (2), hacia lo ambiguo, la proposición (1), lo cual permite abarcar, además de la ubicación precisa (1), específica, también a los x puntos de los alrededores, o incluso de lugares lejanos, como Roma.

Y después (2) es especificada hacia otra nueva proposición interpretada $(3) \neq(1)$ que sí sea verdadera, al incorporar a posteriori a la ubicación o al objeto específico $\neg(1)$ donde el hecho fa vorable acontezca; aunque sea diferente a la ubicación o al objeto exactos correspondientes a (1), a los que se hacía referencia. Y hacemos así verdadera a esa generalización. La cual en realidad también se vuelve falsa si sustituimos su variable por la constante lógica de la especificación (1), que es aquélla que expresa en cada uno de los casos citados la creencia que tiene cada uno de los individuos postulados. Así, (1) es falsa, (3) es verdadera; y (2) es ambigua, y no hay por qué confundirla ni con (1) ni con (3).

\section{Los límites en la justificación de una creencia.}

Parecidamente a los casos citados, Goldman expone la siguiente situación:

Henry está manejando en el campo con su hijo ... "Esa es una vaca," dice Henry, "Ese es un tractor," "Ese es un silo," "Eso es un granero," etc. ... Cada objeto está plenamente a la vista, Henry tiene una excelente visión, y tiene suficiente tiempo para mirar con cuidado razonable, puesto que hay poco tráfico para distraerlo. ... Supóngase que se nos dice que, sin saberlo Henry, el distrito al que ha entrado está lleno de facsímiles de graneros de papel maché ... Estos facsímiles se miran desde el camino exactamente como graneros, pero realmente son solo fachadas ... El objeto que él ve es un establo genuino. Pero si el objeto en ese sitio fuera un facsímil, Henry podría confundirlo con un granero. Dada esta nueva información, estaríamos fuertemente inclinados a retirar el dicho de que Henry sabe que el objeto es un granero. ... Es accidental que Henry tenga razón acerca de que es un granero. Así que retiramos nuestra atribución de que conoce. (Goldman 1976, pp. 772-773. Traducción propia)

Hay aquí otro cambio de constante. Henry se refiere al granero g, y a él remite su creencia. Mas Goldman hace un cambio hacia la variable $\mathrm{x}$, todos los facsímiles del lugar, y luego especifica respecto a cada uno de éstos como constantes $h, i$, j, etc., que cumplen cada uno con ser meros facsímiles, pero exceptuando precisamente a la construcción que viene al caso para la creencia de Henry, que es g.

Hay dos maneras en que se puede justificar una creencia: o por la experiencia, o por ser una verdad lógica.

Los dos casos que expone Gettier pretenden ser creencias justificadas a través de verdades lógicas.

En los demás casos que aquí mencionamos, únicamente es posible justificar la creencia con base en la experiencia. Ahí reside el por qué "No necesitan incluir inferencia alguna".

Existen dos clases de experiencia, la directa y la indirecta. Los casos del espejismo, Platón, la falsa oveja y Henry pueden ser comprobados (justificados o negados) directamente. Basta llegar hasta donde supuestamente estaba el espejismo, voltear a ver al otro corredor claramente, acercase lo suficiente a la falsa oveja, o inspeccionar el granero más minuciosamente. 
Pero también pueden ser justificados como lo plantean los hechos descritos por los autores de estos casos (salvo en el caso del espejismo, pues respecto a los espejismos en el desierto podría creer el sujeto que son frecuentes, y entonces hace falta ver para creer), indirectamente, por inducción, lo noten o no lo noten tales investigadores: sin voltear a ver claramente, sin acercase lo suficiente, o sin mirar más cuidadosamente.

Esto sólo puede ocurrir con base en las experiencias previas, cuando, por ejemplo, se conoce que Sócrates es la persona que frecuentemente acostumbra correr al lado, o que los casos de falsas ovejas son mucho menos frecuentes en el mundo real que el de ovejas verdaderas; o que lo mismo pasa con respecto a los falsos y los verdaderos graneros.

Por el planteamiento de Goldman, Henry no cree que su creencia esté justificada como una verdad lógica. O si lo cree, entonces su creencia no está justificada. Así que Henry únicamente puede creer válidamente que su creencia está justificada por la experiencia. Mas Henry no agota los medios disponibles para la comprobación empírica directa, al no hacer, e.g., un examen más meticuloso. Así, o Henry no cree que su creencia esté justificada como una comprobación directa suficiente; o, si lo cree, entonces su creencia no está justificada.

Sólo le queda a Henry la experiencia indirecta. Y si se llega a preguntar sobre la justificación de su creencia, sólo es válido justificadamente que reconozca que ella está basada en la inducción desde su experiencia previa, al faltarle profundizar en la comprobación directa.

Éste, al que podemos llamar déficit relativo de corroboración directa, es el que se sustituye recurriendo a la inducción, consciente o subconscientemente.

Goldman solo elige los puntos que se acomodan a su argumento, los del campo por el que transita Henry, sin tomar en cuenta todo el universo de graneros que previamente pueda conocer éste, directamente o por referencia, donde lo que priva abundantemente son los graneros reales y no las fachadas que los simulan.

Lo que es accidental, en muy primer término, es que Henry esté pasando por uno de los escasos lugares de su país y del mundo donde predominan los facsímiles. Y tomando en cuenta que su juicio presupone lo que él conoce de la inmensa mayoría de las fachadas de graneros, el que corresponden a construcciones reales, entonces acertó no por azar, sino porque en realidad, por inducción a partir de su conocimiento previo, eso era lo más probable.

Esto último es lo único que puede dar pie al autor para pensar que la creencia de Henry es justificada. Y permitir el no exigirle, para considerarla así, el que se tendría que haber hecho una comprobación empírica directa, una inspección más detallada, fácilmente disponible, del granero.

En este caso Goldman incurre en lo que Tversky y Kahneman llaman un sesgo o prejuicio (bias), una ilusión cognitiva (el de descuido de las tasas de base) (base rates neglect) (vid. Tversky 1982). Al no considerar en su argumento a la frecuencia de base en todo el universo pertinente de lo que sabe Henry acerca de los graneros, para justificar o no la creencia de Henry; sino únicamente a una parte pequeña del mismo universo, la de los falsos graneros en esa zona.

Lo que en este asunto hubiera sido accidental como un hecho, respecto a su universo de conocimiento previo, es que el granero que Henry creía ver hubiera sido un facsímil; que su creencia sobre ese granero en específico no hubiera coincidido con la realidad y, por lo tanto, él estuviera equivocado. 
En síntesis, Goldman sólo puede presuponer implícitamente (para afirmar que existe una creencia justificada) que Henry hace una inducción, y emite su juicio tomando para la tasa de base de ella a su universo de conocimientos previo A. Pero luego, subrepticiamente, cambia de frecuencia de base hacia un universo B, subconjunto propio de A, mucho más limitado; y sobre este cambio, no válido lógicamente, fundamenta su argumento para negar a posteriori que exista conocimiento.

Goldman da el paso del "Existe uno y sólo un universo A" a la generalización sintáctica "Existe algún universo $\mathrm{X}$ " y de ahí a la especificación sustituta "Existe un universo X=B", y así confunde las condiciones necesarias y suficientes para la creencia justificada (las del universo de los conocimientos previos de Henry acerca de los graneros), con las necesarias pero no suficientes (la consideración únicamente de los falsos graneros en esa zona).

\section{Conclusiones.}

Linda Zagzebski establece un procedimiento para construir casos Gettier, el cual, considera, los hace inescapables:

Comience con un caso de creencia falsa justificada (o garantizada). Haga al elemento de justificación (garantía) lo suficientemente fuerte para conocimiento, pero haga la creencia falsa ... debido a algún elemento de suerte. Ahora enmiende el caso, agregando otro elemento de suerte, solo que esta vez haga a la creencia verdadera después de todo. El segundo elemento debe ser independiente del elemento de garantía de manera que el grado de garantía no cambie. (Zagzebski 1994, p. 69. Traducción propia)

Sin embargo, existe adicionalmente una estructura más profunda, falaz, del procedimiento, la oracular, cuyo reconocimiento permite escapar de los problemas Gettier. Y es el paso del "Existe uno y sólo uno" al simple "Existe" y de ahí, sin justificación, al inconsistente cambio de constante, tratando a una oración de creencia como si fuera una oración simple; y confundiendo las condiciones necesarias y suficientes con las necesarias pero no suficientes.

Es decir:

1. Comenzar con una expresión (1) referida a un objeto, un hecho, un lugar o un universo de discurso determinado, conteniendo una constante lógica c referida a cualquiera de esos aspectos, con sus particulares condiciones necesarias y suficientes para la creencia justificada.

2. Generalizar la misma expresión hacia una nueva expresión (2), usando una variable lógica $\mathrm{x}$ en lugar de la constante, suprimiendo alguna de aquellas condiciones necesarias. 3. Volver a interpretar, sin justificación lógica, la expresión general (2), con una nueva constante lógica d distinta y contradictoria de c, refiriéndose a un objeto, un hecho, un lugar, o un universo específico que se adecue a posteriori a la situación que realmente ocurra; pero que no cumpla con alguna condición necesaria suprimida. Generando así una nueva expresión interpretada (3), pero confundiendo indebidamente (3) con (2).

Eso convierte al razonamiento tipo Gettier en una argumentación de tipo sibilino, lógicamente inconsistente.

\section{Bibliografía.}

(1) Ayer, A. J., 1956, The Problem of Knowledge (London: Macmillan). 
(2) Boh, Ivan, 1985, "Belief, Justification and Knowledge: Some Late Medieval Epistemic Concerns", en Journal of the Rocky Mountain Medieval and Renaissance Association, 6, pp. 87-103. Citado en Ichikawa 2018.

(3) Chisholm, Roderick, 1957, Perceiving: A Philosophical Study (New York: Cornell University Press). 1977, Theory of Knowledge, Prentice Hall, New Jersey.

(5) Dretske, Fred, May 1971, "Conclusive reasons", en Australasian Journal of Philosophy. 49 (1), pp. 1-22. doi:10.1080/00048407112341001.

(6) Dreyfus, George B.J, 1997, Recognizing Reality: Dharmakirti's Philosophy and its Tibetan Interpretations (Albany, New York: Suny Press). Citado en Ichikawa 2018.

(7) Gettier, Edmund L., 2013, "¿Una creencia verdadera justificada es conocimiento?", en Disputatio. Philosophical Research Bulletin 3, pp. 185-193.

www.disputatio.eu.

(8) Goldman, Alvin I., 1976, "Discrimination and Perceptual Knowledge", en The Journal of Philosophy, 73(20), pp. 771-791. doi:10.2307/2025679.

(9) Heródoto de Halicarnaso, 1977, Historias, Libro I (Madrid: Ed. Gredos).

(10) Ichikawa, Jonathan, Jenkins y Matthias Steup, Verano 2018, "The Analysis of Knowledge", en The Stanford Encyclopedia of Philosophy, Edward N. Zalta (ed.). $\mathrm{URL}=\langle$ https://plato.stanford.edu/achives/sum2018/entries/knowledge-analysis/>.

(11) Lycan, William. G., 2006, "On the Gettier Problem problem", en Epistemology Futures, S. Hetherington (ed.) (Oxford: Oxford University Press), pp. 148-168.

(12) Nozick, Robert, 1981, Philosophical Explanations (Cambridge: Harvard University Press).

(13) Platón, 2005, Menón, en Diálogos II (Madrid: Ed. Gredos).

(14) Platón, 2015, Teeteto, en Diálogos V (Madrid: Ed. Gredos).

(15) Russell, Bertrand, 1973, "Sobre el denotar", en Simpson, Thomas M. ed., Semántica filosófica: problemas y discusiones (Buenos Aires: Siglo XXI editores), pp. 29-48.

(16) Tversky, Amos y Daniel Kahneman, 1982, "Evidential impact of base rates", en Tversky, Kahneman, y P. Slovic, ed., Judgment under uncertainty: Heuristics and Biases (New York: Cambridge University Press).

(17) Zagzebski, Linda, 1994, "The Inescapability of Gettier Problems", en The Philosophical Quarterly, 44 (174): 65-73, Blackwell Publishing.

doi:10.2307/2220147. 